\section{Legal leverage}

Tony Zigmond's editorial about 'leverage' in English mental healthcare helps to demonstrate the promotion of coercion without appeal (as may be even more common in the USA) resulting from an apparent contradiction in local mental health law:

'Even when taking medication is a condition attached to a conditional discharge (from a restriction order, Mental Health Act 1983) or community treatment order, it would be unlawful to recall the individual solely because the individual decided to refuse the medication.' 1

Presumably at the time of conditional discharge (including the requirement that they continue prescribed medication) the person is well enough to cope with the requirement. They are coerced (by stated conditions), hopefully, because it has been demonstrated that without medication they are highly likely to become unwell again.

So what is different about the situation where the person is out of hospital and stops their medication, that they cannot be legally coerced to take it? Has the person changed in some way when they face a similar clinical risk?

Is it better to have a legal provision for this coercion outside the hospital, with all the necessary legal safeguards and reviews required (as under the Australian New South Wales Mental Health Act 2007) or to await decompensation and have caring people desperately trying to forestall such an event, possibly with illegal (and possibly inappropriate) 'leverage' pressures?

The article does not address the often characteristic accompanying distress to the person and their network when a person decompensates into more disorganised psychosis. Perhaps 'he died with his rights on'.

In the Australian New South Wales Act, intervention requires not only the 'least restrictive' option, but also the 'continuing condition' of a mentally ill person and risk of ANY harm. In other words, if the history indicates a chronic illness and the likelihood of decompensation off medication, this must be considered, even it the person is apparently getting by at the time.

Perhaps failure to initiate sensitive intervention, including coercion as necessary, with legal safeguards and appeal capacity, in such circumstances, is negligence. Such intervention may also be regarded as a 'loving act'.

To say that a person has the capacity to make a decision does not determine the quality or appropriateness of that decision.

To say that a person is 'capacitous' because they are capable of apparently understanding their situation (and so 'having the capability of making decisions about their own treatment') and have 'insight' is to leave open much definitional argument, but it is not appropriate as the sole determinant of non-intervention in a situation of carefully determined clinical risk to the person and the legitimate distress of others.

In asking 'Who should have the final say?', Zigmond seems to assume that this question is resolved by choosing either the patient or the psychiatrist. In matters of coercion it is the law that has the final say and at its best it seeks widely and wisely for an answer hence tribunals. Hence also the need for good law.

It may be that the (statistical numbers) rise in coercion in psychiatric practice is necessary, but dealing with doubts about its desirability is not best met by placing difficulties in the way of people getting treatment they need to live their lives most fulfillingly and with others. Rather, the preferred emphasis may be for more careful review, in particular cases, of the necessity of coercion and of prescribing choices.

1 Zigmond T. Pressures to adhere to treatment: observations on 'leverage' in English mental healthcare. Br J Psychiatry 2011; 199: 90-1.

Donald Scott-Orr, Port Macquarie, New South Wales, Australia Email: dnsf@bigpond.com

doi: $10.1192 / \mathrm{bjp} .200 .1 .81$
Coercion, compulsion, adherence, compliance, persuasion and like terms need to be in our clinical consciousness, as recently articulated in the editorial by Zigmond in relation to an English study on leverage. ${ }^{1,2}$ The suggestion that resolving the practice of leverage/coercion is best left to patient choice assumes that there is a lot of coercion going around, albeit erringly. His assumption that other branches of medicine are devoid of similar practice is flawed and incorrect. Removal of children who are obese from their parents, denial of driving rights to individuals with epilepsy who choose to drive and the mandatory revelation of HIV status to partners by reluctant spouses are but few examples of similar coercion in other branches of medicine. It is worth noting that, in these examples and in the examples of mental illness, focusing only on the patient's choice narrows the implications of those choices as they affect others. It also takes away from physicians the collective role they play to the society at large. The following reasons are why, at the time we are trying to allow voluntary participation in treatment decisions, we must guard against amplifying and magnifying 'choice'.

First, choice is shaped and essentially dependent on insight. Correlates of insight are no longer restricted to anosognosia-like views or neuropsychological dysfunction based on injuries to frontal, parietal or temporal lobes. Volumetric reductions in several cortical regions evinced by neuroimaging studies are well documented in chronic schizophrenia and first-episode psychosis. ${ }^{3,4}$ Consequently, lack of insight, unawareness of illness and the need for treatment can no longer be relegated to the domain of choice. Those involve a network of brain structures affected by the disorder. Even the law recognises this in assigning the 'but for mental disorder' designate in various medico-legal criteria.

Second, medico-legal provisions of treatment are unfortunately driven by public reactions to failures in the mental health system. The law is then made and takes precedence in determining the acceptable level of risk to which the society should be exposed. This is known as 'where the public peril begins..$^{5}$ In recognition of the implications of the choices made for and against treatment, the tension between autonomy and beneficence has not stopped being the most contentious of ethical quagmires. A broader perspective is in order beyond choice.

Third, the rates of coercion cannot categorically be said to be increasing, as opined by Zigmond. To modify the perceived alarming statistics he referenced, other relevant factors should be recognised alongside the increasing number of formal compulsory hospital admissions (1987-2010). The population of English society has not only increased over the past three decades, it has become more diverse with migrants who affect rates of mental disorder as well as the potential for coercion. The tolerance for mental illness and societal acceptance is changing. Community support for patients, a by-product of economic prosperity, has dwindled, ${ }^{6}$ contributing to coercive approaches from both family and practitioners. Although litigation of practitioners is lower in the UK than in the USA, it is nonetheless relevant in determining physicians' attitudes towards voluntariness. ${ }^{7,8}$

Finally, when the treatment of our patients considers the past and the future, progress in reducing stigma necessitates the use of leverage in some situations. The high rates of the use of leverage have been suggested as a good thing. The Americans have experienced a 'before and after' paradigm of reducing leverage. 'Rotting with their rights on' was not only a title of scientific publications but was the terminology used to describe the repercussions. The UK should learn from this that there are tragic and costly consequences in focusing only on choice. ${ }^{9}$ Despite decades of that experiment in the USA, the opinion to use leverage as a positive tool still exists. ${ }^{10}$ 
1 Zigmond T. Pressures to adhere to treatment: observations on 'leverage' in English mental healthcare. Br J Psychiatry 2011; 199: 90-1.

2 Burns T, Yeeles K, Molodynski A, Nightingale H, Vazquez-Montes M, Sheehan $K$, et al. Pressure to adhere to treatment ('leverage') in English mental healthcare. Br J Psychiatry 2011; 199: 145-50.

3 Buchy L, Ad-Dab'bagh Y, Malla A, Lepage C, Bodnar M, Joober R, et al. Cortical thickness is associated with poor insight in first-episode psychosis. J Psychiatr Res 2011; 45: 781-7.

4 Buchy L, Czechowska Y, Chochol C, Malla A, Joober R, Pruessner J, et al. Toward a model of cognitive insight in first-episode psychosis: verbal memory and hippocampal structure. Schizophr Bull 2010; 36: 1040-9.

5 Buchner F, Firestone M. Where the public peril begins: 25 years after Tarasoff. J Leg Med 2000; 21: 25.

6 Becker T, Kilian R. Psychiatric services for people with severe mental illness across western Europe: what can be generalized from current knowledge about differences in provision, costs and outcomes of mental health care? Acta Psychiatr Scand Suppl 2006; 429: 9-16.

7 Glancy DR, Glancy GD. The case that has psychiatrists running scared: Ahmed v. Stefaniu. J Am Acad Psychiatry Law 2009; 37: 250-6.

8 Glancy GD, Chaimowitz G. Tarasoff warnings result in criminal charges. J Am Acad Psychiatry Law 2003; 31: 524-5.

9 Gutheil TG. In search of true freedom: drug refusal, involuntary medication, and 'rotting with your rights on'. Am J Psychiatry 1980; 137: 327-8.

10 Kress K. Rotting with their rights on: why the criteria for ending commitment or restraint of liberty need not be the same as the criteria for initiating commitment or restraint of liberty, and how the restraint may sometimes justifiably continue after its prerequisites are no longer satisfied. Behav Sci Law 2006; 24: 573-98.

Mansfield Mela, Department of Psychiatry, College of Medicine, University of Saskatchewan, Canada. Email: mansfieldmela@gmail.com

doi: 10.1192/bjp.200.1.81a

Author's reply: Thank you to Drs Scott-Orr and Mela for their interest. It seems to me that there are two issues here. First, should the law be discriminatory between patients with a physical illness and those with a mental illness? I think not and I'm pleased to say the United Nations Convention on the Rights of Persons with Disabilities (2006), to which the UK is a signatory, supports this view. The convention obligates States to (among many other things) 'take all appropriate measures, including legislation, to modify or abolish existing laws, regulations, customs and practices that constitute discrimination against persons with disabilities'. To explain this further, the UN High Commissioner for Human Rights said, ${ }^{1}$

'Legislation authorizing the institutionalization of persons with disabilities on the grounds of their disability without their free and informed consent must be abolished... This should not be interpreted to say that persons with disabilities cannot be lawfully subject to detention for care and treatment or to preventive detention, but that the legal grounds upon which restriction of liberty is determined must be de-linked from the disability and neutrally defined so as to apply to all persons on an equal basis.

Second, should the law (for everyone) favour patient autonomy, medically determined best interest or a mixture?

In other words, either everyone, with the capacity to make the decision, should be permitted to 'die (or rot) with their rights on' or nobody should. Or the authority to overrule capacitous refusal could be based on a neutral factor such as risk to other people. It should not be dependent on the stigma associated with certain terminology (a mental illness diagnosis).

1 United Nations. Annual Report of the United Nations High Commissioner for Human Rights and Reports of the Office of the High Commissioner and the Secretary-General: A/HRC/10/48, 26 January 2009. United Nations, 2009.

Tony Zigmond, Newsam Centre, Leeds LS14 6WB, UK.

email: azigmond@doctors.net.uk

doi: 10.1192/bjp.200.1.82

\section{Family psychoeducation for major depression: randomised controlled trial}

The paper by Shimazu et al ${ }^{1}$ adds robustness to already existing evidence for the role of family psychoeducation in psychiatric disorders. The study has sound methodology (i.e. randomised controlled trial) with adequate masking, in addition to being the first ever study to examine the effect of family psychoeducation for major depressive disorder. The authors describe the possible limitations of the study honestly. A source of funding (Grant-inAid for Scientific Research, Ministry of Health, Labour and Welfare, 2004) is also mentioned. However, there are some issues which should be further looked into. The aim was to examine family psychoeducation in the maintenance treatment of depression.

However, the patients included were either on continuation or maintenance treatment. Patients who are in partial remission cannot be considered as being in a continuation/maintenance phase. ${ }^{2}$ Also it was not mentioned how many patients had single or recurrent episodes (patients with single episodes need not receive maintenance phase treatment). Any other psychiatric comorbidity (substance misuse or personality disorder) in the participants was not mentioned, even though it has treatment implications. The health status and intellectual functioning of the primary family member included in the study was not mentioned, although these might compromise their active participation in psychoeducation sessions. The authors are silent on the ethical clearance of the study. For four caregivers psychoeducation sessions were done in the individual's home and not in group sessions, which were not included in the final analysis and not part of the methodology mentioned - this could also have had an effect on the efficacy of the study. Remission was defined by the authors as a Hamilton Rating Scale for Depression (HRSD) score $<6$, but the normal score is mentioned as $<7$. $^{3}$ The authors have not mentioned the reason for keeping a low score of HRSD in the study. Last, it could be a printing mistake, in the last line of Table 1 it is mentioned that high expressed emotion (as per FMSS) was seen in seven patients in the intervention group and none in the control group, but the results mention that it is seen in six patients in the intervention group and ten in the control group.

1 Shimazu K, Shimodera S, Mino Y, Nishida A, Kamimura N, Sawada K, et al. Family psychoeducation for major depression: randomised controlled trial. Br J Psychiatry 2011; 198: 385-90.

2 American Psychiatric Association. Practice Guideline for the Treatment of Patients with Major Depressive Disorder, Third Edition. APA, 2010.

3 Blacker D. Psychiatric rating scales. In Kaplan and Sadock's Comprehensive Textbook of Psychiatry, Ninth Edition, Volume 1 (eds BJ Sadock, VA Sadock, P Ruiz): 1047. Lippincot Williams and Wilkins, 2009.

Bichitra N. Patra, Department of Psychiatry, Postgraduate Institute of Medical Education and Research, Chandigarh - 160012, India. Email: patrab.aiims@gmail.com; B. N. Subodh, Department of Psychiatry, Postgraduate Institute of Medical Education B. N. Subodh, Departh
and Research, India

doi: 10.1192/bjp.200.1.82a

Author's reply: We thank Patra \& Subodh for their interest in and their very thorough reading of our study. ${ }^{1}$ Most of their questions are factual ones and we are grateful that we have been given an opportunity to clarify them. First, whether to call further treatment of patients in partial or full remission after the fully syndromatic episode, as in our study, continuation/ maintenance treatment is a terminological issue and not a medically substantive one. And we think our usage of the terms is in consonance with the majority of psychiatrists of the world, as for example done by Paykel et al in their famous study of cognitive therapy to prevent relapse after acute episode of major depression. $^{2}$ 